\title{
EDITORIAL
}

\section{The Democratization of CRISPR}

Rodolphe Barrangou

By many measures, CRISPR*-based technologies and their applications have taken over the world in the past few years, and the CRISPR era is upon us. Much like cloning and PCR in prior decades, CRISPR stands to become a generation-defining technology. Indeed, as illustrated on the cover of this issue and discussed in the Perspective by LaManna and Barrangou on page 205, researchers continue to advance, develop, and implement a wide array of CRISPR tools, which are now being circulated globally in real time by Addgene. Embraced by commercial entities and fueled by motivated investors, the commercialization of CRISPR is next.

The goal of making CRISPR openly available, regardless of location, political, or regulatory consideration, epitomizes the mission of Addgene, a nonprofit organization aimed at empowering academic laboratories by granting access to cutting-edge technologies and advance science. This is a noteworthy departure from ongoing intellectual-property disputes-and a refreshing digression from some perplexing steep licensing fees charged by not-for-profit academic institutions that hold rights to CRISPR tools.

The numbers alone reflect the Addgene-enabled pervasiveness of CRISPR, not just in North America, Europe, and Asia, but also in many third world countries. CRISPR is no longer an exclusive technology but rather universalinternationally, culturally, and socially. For example, new applications of CRISPR in a diagnostic context offer great potential in territories at risk of disease outbreaks with limited resources (see the First Cut article by Karl Petri and Vikram Pattanayak, page 209). Naturally, governments and the public are actively evaluating how this technology should be managed; their opinions and actions will have widespread impact on both science and society.

Public opinion has proven to be a powerful modulator of scientific progress, leading to long-lasting repercussions for many countries. As always, one hopes that scientifically informed decisions will be made, but history and recent events remind us that this is not always the case, given the public perception about science, use of misguided

*Clustered Regularly Interspaced Short Palindromic Repeats. terms such as "frankenfood" and "designer babies" by the media, and historical shortcomings displayed by the agriculture (Ag) industry with regard to public relations, communications, and stewardship. Yet, there is hope. While much focus has been on the science, we should be mindful of the people driving these efforts. Indeed, scientists spend decades training and preparing for such opportunities and dedicate most of their lives to solving problems and creating solutions. The recent "Unite to Cure" conference at the Vatican signaled a new chapter for CRISPR acceptance and stewardship (see the First Cut by Davies on page 213) and extends a crucial dialogue about ethical implications and societal engagement.

Already, CRISPR is transcending not just scientific and economic boundaries for medicine and agriculture, but also religious, ethical, and societal frontiers. Besides the well-documented therapeutic implications, notwithstanding recent Food and Drug Administration clarification requests, many signs already point to tremendous benefits pending in agriculture (see the First Cut by Willmann on page 211). Arguably, agriculture is poised to win the CRISPR race: teams of scientists in academia and industry, including "big Ag" and several new start-up companies, are harnessing next-generation breeding of crops and livestock to help feed the world. The benefits comprise classical targets such as increased yield, pest management, and drought resistance to address the food gap for our rapidly expanding population. Efforts increasingly encompass sustainability, with improved water usage and efficient land management receiving critical attention. We need more food, but also a safer and healthier supply chain, globally.

Our ability to bring CRISPR to the people hinges on both supportive adjudication by regulatory agencies and legislators as well as public acceptance of science and technology. The challenges are formidable given skepticism of "big Ag" along with confusion and uncertainty over what CRISPR actually entails. Actually, there is an opportunity for both sides to discuss how to best regulate genome editing to encourage support rather than constrain the advancement and exploitation of these beneficial technologies. 
A major milestone was the March 2018 announcement by the U.S. Department of Agriculture stating that it will not regulate genetically edited plants that recapitulate traditional breeding results is encouraging, but this is only the beginning. Concerted efforts by multiple communities and stakeholders are underway to formalize the regulatory landscape with input from academics and PR experts. Those addressing the food gap and harnessing CRISPR-based technologies to ensure a healthier and more sustainable food supply must better convey how crit- ical, impactful, and noble their efforts are. Unity, caution, transparency, and engagement constitute the path forward. A series of recent and forthcoming meetings will define the ability of CRISPR to make progress in the short term.

For CRISPR to realize its obvious potential, the scientific community must strive to share its progress with all stakeholders: we all need to do a better job at communicating about science in general and telling and spreading the CRISPR story in particular. It is unclear if and how quickly we will get there. 